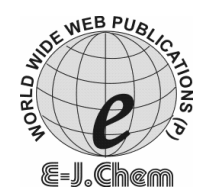

http://www.e-journals.net
ISSN: 0973-4945; CODEN ECJHAO

E-Journal of Chemistry 2009, 6(1), 113-119

\title{
Variational Principle Techniques and the Properties of a Cut-off and Anharmonic Wave Function
}

\author{
A. N. IKOT, L. E. AKPABIO, K. ESSIEN, E. E. ITUEN and I. B. OBOT* \\ Department of Physics, University of Uyo, Nigeria. \\ *Department of Chemistry, University of Uyo, Nigeria. \\ proffoime@yahoo.com
}

Received 23 March 2008; Revised 19 May 2008; Accepted 10 July 2008

\begin{abstract}
The variational principles are very useful analytical tool for the study of the ground state energy of any dynamical system. In this work, we have evaluated the method and techniques of variational principle to derive the ground state energy for the harmonic, cut-off and anharmonic oscillators with a ground state wave function for a one-body Hamiltonian in three dimensions.
\end{abstract}

Keywords: Harmonic oscillator, Cut-off harmonic oscillator, Anharmonic oscillator, Variational method.

\section{Introduction}

Since most problems in Physics and Chemistry cannot be solved exactly, one resort to the use of approximation methods. The two methods used commonly in quantum mechanics are the perturbation theory and variational method. The basic idea of the perturbation theory involves the splitting of the Hamiltonian into two pieces - the unperturbed and perturbation. This theory is useful when there is a small dimensionless parameter in the said problem called the coupling constant and the system becomes solvable when this parameter is set to zero. Thus the perturbation theory gives correction as an infinite series of terms in powers of the perturbation parameter which becomes smaller for a well-behaved system.

On the other hand, the variational method is useful for the study of the ground state system even though it is not useful for the study of the excited states of a system. The basic idea of the variational method lies in the choice of a trial wave function for the problem at hand; which consists of some adjustable parameters usually called the variational parameters. These variational parameters are usually adjusted until the energy of the trial wave function is minimized. Once this is done, the resulting trial wave function and its corresponding eigen values are variationally approximate to the exact wave function and energy. 
The variational principle and simple properties of the ground state wave function with simple potential had been investigated ${ }^{1}$. They show that the ground state wave function can be taken to be real and non-negative and that it can not be degenerate where their parity and angular momentum of this ground state wave function has also been analysed ${ }^{1}$. The properties of the ground state wave function with simple and complex potential had been examined by different authors ${ }^{2-4,11-14}$. The variational perturbation theory in terms of path integral approach had been analyzed ${ }^{5-7}$ with different potentials.

In this paper, we follow the approach ${ }^{1}$ and extend their application to a cut-off harmonic and the anharmonic oscillator.

\section{Fundamentals of variational principle}

The ground state wave function has the lowest-energy eigen value of a given system.

$$
H\left|\psi_{0}\right\rangle=E_{0}\left|\psi_{0}\right\rangle
$$

and any other states have higher energy eigen values

$$
H\left|\psi_{n}\right\rangle=E_{n}\left|\psi_{0}\right\rangle
$$

where $\left.E_{n}\right\rangle E_{0}$. For the Hamiltonian which cannot be solve for eigen states and eigen values exactly, we use a trial wave function or an ansatz as

$$
\Phi=\sum_{n=0}^{\infty} C_{n} \psi_{n}
$$

The ansatz is exact if $C_{0}\left\langle 1\right.$ and $\mathrm{C}_{\mathrm{n}}=0$ for all $n \neq 0$ and the normalization requires that

$$
\left|C_{0}\right|^{2}+\sum_{n \neq 0}\left|C_{n}\right|^{2}=1
$$

The approximate energy or the expectation value with this ansatz is

$$
\langle\phi|\hat{H}| \phi\rangle=\left|C_{0}\right|^{2} E_{0}[\phi]+\sum_{n \neq 0}\left|C_{n}\right|^{2} E_{n}[\phi] \text { or } E_{0}[\phi]=\langle\phi|\hat{H}| \phi\rangle
$$

This equation holds if the ansaltz is normalized but if the wave function is not normalized, (5) becomes

Substituting (3) into (6) yields

$$
E_{0}[\phi]=\frac{\langle\phi|\hat{H}| \phi\rangle}{\langle\phi \mid \phi\rangle}
$$

$$
E_{0}[\phi]=\frac{\sum_{i j} C_{i}{ }^{*} C_{j}\left\langle\psi_{i}|H| \psi_{j}\right\rangle}{\sum_{i j} C_{i}{ }^{*} C_{j}\left\langle\psi_{i} \mid \psi_{j}\right\rangle}
$$

Since the functions $\psi_{j}$ are the exact eigen functions of $\hat{H}$, we can write

$$
\begin{array}{r}
\hat{H} \psi_{j}=\in_{j} \psi_{j} \text { and obtain } \\
E_{0}[\phi]=\frac{\sum_{i j} C_{i}{ }^{*} C_{j} \in_{j}\left\langle\psi_{i} \mid \psi_{j}\right\rangle}{\sum_{i j} C_{i}{ }^{*} C_{j}\left\langle\psi_{i} \mid \psi_{j}\right\rangle}
\end{array}
$$

On subtracting $\in_{0}$ from both sides of (9) we obtain that

$$
E_{0}[\phi] \geq \epsilon_{0}
$$

This implies that the trial wave functions is always greater than or equal to the exact ground state energy $\epsilon_{0}$. With this observation one look for a ground state wave function by introducing variational parameters to the ansatz $\left|\phi\left(\lambda_{1}, \ldots . \lambda_{2} \ldots . . \lambda_{n}\right)\right\rangle$ and calculating the 
energy expectation value result

$$
\left\langle\hat{E}\left(\lambda_{1}, \lambda_{2} . .\right)\right\rangle=\left\langle\phi\left(\lambda_{1}, \lambda_{2} \ldots\right)|\hat{H}| \phi\left(\lambda_{1}, \lambda_{2} \ldots\right)\right\rangle
$$

and looking for as low expectation value as possible by minimizing (11) leads to

$$
\frac{\partial\langle E\rangle}{\partial \lambda_{1}}=\frac{\partial\langle E\rangle}{\partial \lambda_{2}}=0
$$

The success of variational method depends on a good trial wave function with the good set of parameters. This is the fundamental of the variational method.

\section{Reality and position definiteness of the wave function}

The stationary Schrödinger equation for a spinless particle in three dimensions moving under the influence of a potential $\mathrm{V}(\mathrm{r})$ is given as ${ }^{1}$

$$
H \psi(r)=-\frac{\hbar^{2}}{2 m} \nabla^{2} \psi(r)+V(r) \psi(r) \quad=E \psi
$$

Where $\mathrm{H}$ is the Hamiltonian of the system and $\mathrm{E}$ is the corresponding eigen values. Due to the spontaneous symmetry breaking occurs which may lead to degeneracy in the groundstate wave function the velocity dependent potential is not considered in this approach as pointed out by ${ }^{8}$.

The trial wave function for the system is written as

$$
|\psi(r)\rangle=|f(r)\rangle e^{i x(r)}
$$

Where assumption is made by the fact that the Hamiltonian in (13) has at least one bound state and the quantity $f(r)$ and $\chi(r)$ are single and real-valued function. Using (14), we calculate the expectation value of the potential energy as

$$
\left\langle E_{v}\right\rangle=\frac{\langle\psi|V| \psi\rangle}{\langle\psi \mid \psi\rangle}
$$

Also using (14) and (15) lead to

$$
\left\langle E_{v}\right\rangle=\frac{\langle f(r)|V(r)| f(r)\rangle}{\langle f(r) \mid f(r)\rangle}
$$

which shows that the potential energy is unaffected by the complex parameter $\chi(r)$ of the wavefunction.

The kinetic energy of the system is calculated via the use of the Jackson Feenberg identity ${ }^{9}$ taking into consideration the anti-hermetian nature of the gradient operator ${ }^{1}$, thus

$$
\left\langle E_{T}\right\rangle=\frac{\langle\psi|T| \psi\rangle}{\langle\psi \mid \psi\rangle} \quad=-\frac{\hbar^{2}}{8 m} \int \frac{d^{3} r\left[\left(\nabla^{2} \psi^{*}\right) \psi+\psi^{*}\left(\nabla^{2} \psi\right)-2\left(\nabla \psi^{*}\right) \cdot(\nabla \psi)\right]}{\int d^{3} r \psi^{*} \psi}
$$

Now taking the gradient of (14) results

$$
\nabla \psi(r)=e^{i \chi(r)}[\nabla f(r)+i f(r) \nabla \chi(r)]
$$

and by taking its complex conjugate, equation(18) becomes

$$
\nabla \psi^{*}(r)=e^{-i \chi(r)}[\nabla f(r)+i f(r) \nabla \chi(r)]
$$

By virtue of the two relations (18) and (19), one can easily show that the following relationship holds, 


$$
\begin{aligned}
\left(\nabla^{2} \psi^{*}\right) \psi+\psi^{*}\left(\nabla^{2} \psi\right) & =2 f(r) \nabla^{2} f(r)-2 f^{2}(r)(\nabla \chi(r))^{2} \text { and } \\
\left(\nabla \psi^{*}\right) \cdot \nabla \psi & =(\nabla f(r))^{2}+f^{2}(r)(\nabla \chi)^{2}
\end{aligned}
$$

With equation (17), (20) and (21), we obtain the expectation value of the kinetic energy operator as

$$
\left\langle E_{T}\right\rangle=\frac{\langle\psi|T| \psi\rangle}{\langle\psi \mid \psi\rangle}=\frac{\hbar^{2}}{2 m} \frac{\int d^{3} r\left[(\nabla f(r))^{2}\right]}{\int d^{3} r f^{2}(r)}+\frac{\hbar^{2}}{2 m} \frac{\int d^{3} r f^{2}(r)(\nabla \chi(r))^{2}}{\int d^{3} r f^{2}(r)}=\left\langle E_{T}^{\delta}\right\rangle+\left\langle E_{T}^{\alpha}\right\rangle
$$

The total Hamiltonian of the system can now be written as

$$
\langle H\rangle=\left\langle E_{T}\right\rangle+\left\langle E_{v}\right\rangle=\langle\boldsymbol{E}\rangle+\left\langle E^{\rangle}\right\rangle+\left\langle E_{v}\right\rangle
$$

When compares with the expectation value of the potential energy operator, we observed that the expectation value of the kinetic energy depends on both the real and complex function $f(r)$ and $\chi(r)$ as shown in (22). As noted by ${ }^{1}$, since $\left\langle E_{T}\right\rangle \geq 0$ one can always decrease the energy associated with the ansatz (14) by setting

$$
\nabla \chi(r)=0
$$

this implies making the phase factor a constant. As a consequence, the setting of $\nabla \chi(r)$ to zero in the wave function does not affect the result. Therefore, one is justified by choosing as the condition $\chi=0$ and wave function should admit the representation

$$
|\psi(r)\rangle=f(r) \geq 0
$$

for the ground state wave function, which indicates that the wave function is real and does not change sign.

As noted before, this wave function does not hold for the velocity-dependent potential. Other consequences like the non degeneracy, the angular momentum and the parity of the wave function have been explicitly exposed ${ }^{1}$.

\section{Applications to the harmonic oscillators}

In this section, we will use the technique of variational methods to calculate the ground eigen value of the Harmonic oscillators in three dimensions and compare the minimized energy with the exact result and then extend the applications to the cut-off and the anharmonic oscillators.

\section{Harmonic oscillator}

Here, we consider a system with the Hamiltonian

$$
H=\frac{p^{2}}{2 m}+m \omega^{2} r^{2}
$$

We assume the ansatz for the ground state wave function in the form

$$
|\phi\rangle=N e^{-\alpha r^{2} / 2}
$$

and calculate the minimum energy and then compare the result with the exact result given by

$$
\begin{aligned}
\psi(r) & =\left(\frac{m \omega}{\pi \hbar}\right)^{3 / 4} e^{-m \omega^{2} r^{2} / 2 \hbar} \\
\langle E\rangle & =3 / 2 \hbar \omega
\end{aligned}
$$

where $\omega$ is the oscillator frequency. Normalizing (27) and comparing with (28) yields

$$
N=\left(\frac{\alpha}{\pi}\right)^{3 / 4}
$$


While the total energy for the trial wave function in terms of the variational parameter $\alpha$ is

$$
E(\alpha)=\frac{\langle\phi|\hat{H}| \phi\rangle}{\langle\phi \mid \phi\rangle} 3 / 2 \hbar^{2} / m \alpha+3 / 4 m \omega^{2} \alpha^{-1}
$$

On minimizing $E(\alpha)$ with respect to $\alpha$ results

$$
\frac{d E}{d \alpha}=\frac{3}{4} \hbar^{2} / m-\frac{3}{4} m \omega^{2} / \alpha^{2}=0 \text { or } \alpha=\frac{m \omega}{\hbar}
$$

We identify the length scale of the Harmonic oscillator as $\lambda=\sqrt{\frac{\hbar}{m \omega}}$ and on substituting $\alpha$ into (30) yield the same value as the exact result given by (28).

\section{The cut-off harmonic oscillator}

We now consider a system with the Hamiltonian

$$
\begin{gathered}
H=\frac{p^{2}}{2 m}+V(r) \\
\text { Where } V(r)=\left\{\begin{array}{l}
\frac{m \omega^{2} r^{2}}{2}-\frac{a^{2}}{r}, \\
\infty
\end{array}\right.
\end{gathered}
$$

We follow the approach ${ }^{3}$ and use as trial function

$$
|\psi\rangle=\left(\frac{m \omega}{\hbar}\right)^{3 / 4} \frac{2 r}{\pi^{1 / 4}} e^{-\frac{m \omega^{\prime} r^{2}}{2 \hbar}}
$$

where $\omega^{\prime}$ is the variational parameter using the ansatz (32), we estimate the ground state energy of the system as

$$
E\left(\omega^{\prime}\right)=\frac{3}{4} \hbar \omega^{\prime}\left(1-\frac{8 m a^{2}}{3 \hbar^{2}}\right)+\frac{3}{4} \frac{\hbar \omega^{2}}{\omega^{\prime}}
$$

Also by minimizing (34) as

$$
\begin{aligned}
\frac{\partial E\left(\omega^{\prime}\right)}{\partial \omega^{\prime}} & =0 \\
\text { we one obtain } \omega^{\prime} & =\frac{\omega}{\sqrt{1-\frac{8 m a^{2}}{3 \hbar^{2}}}}
\end{aligned}
$$

and on substituting (36) into (34) yields

$$
E\left(\omega^{\prime}\right)=\frac{3}{4} \hbar \omega\left(1-\frac{8 m a^{2}}{3 \hbar^{2}}\right)^{-1 / 2}+\frac{3}{4} \hbar \omega\left(1-\frac{8 m a^{2}}{3 \hbar^{2}}\right)^{1 / 2}
$$

Taylor expanding to first order the arguments in the square root of (37) leads to

$$
E\left(\omega^{\prime}\right)=\frac{3}{4} \hbar \omega e^{\frac{8 m a^{2}}{3 \hbar^{2}}}+\frac{3}{4} \hbar \omega e^{-\frac{8 m a^{2}}{6 \hbar^{2}}}=\frac{3}{4} \hbar \omega\left[e^{x}+e^{-x}\right]
$$

We can recast (38) in the form

$$
E\left(\omega^{\prime}\right)=\frac{3}{4} \hbar \omega \cosh \left(\frac{8 m a^{2}}{6 \hbar^{2}}\right)
$$


Where $X=\frac{8 m a^{2}}{6 \hbar^{2}}$ in (37). When $a \rightarrow 0$ in (39) yields the ground state energy $E\left(\omega^{\prime}\right)=\frac{3}{4} \hbar \omega$ as was expected

\section{The anharmonic oscillator}

The Hamiltonian of this system is defined as

$$
H=\frac{p^{2}}{2 m}+\frac{1}{2} m \omega^{2} r^{2}+\alpha \hbar \omega\left(\sqrt{\frac{m \omega}{\hbar}} x\right)^{4}
$$

Again, we assume the trial function of the form

$$
\mid \psi\left(x, \omega^{\prime}\right\rangle=\frac{1}{\pi^{1 / 4}}\left(\frac{m \omega}{\hbar}\right)^{3 / 4} e^{-m \omega^{\prime} r^{2} / 2 \hbar}
$$

Evaluating the ground state energy with the trial function (41) results ${ }^{3}$

$$
E\left(\omega^{\prime}\right)=\frac{\hbar \omega^{\prime}}{4}+\frac{\hbar \omega^{2}}{4 \omega^{\prime}}+\frac{3 \alpha \hbar \omega^{3}}{4 \omega^{2}}
$$

and on minimizing (42) yields

$$
\begin{gathered}
\frac{\partial E\left(\omega^{1}\right)}{\partial \omega^{1}}=\frac{\hbar}{4}-\frac{\hbar \omega^{2}}{4 \omega^{2}}-\frac{6 \alpha \hbar \omega^{3}}{4 \omega^{\prime 3}} \\
\frac{\omega^{3}}{\omega^{3}}-\frac{\omega^{\prime}}{\omega}-6 \alpha=0
\end{gathered}
$$

If one substitutes $\omega^{1}=\omega$ in (41) then the ground state energy becomes

$$
E\left(\omega^{\prime}\right)=\frac{\hbar \omega}{2}+\frac{3 \alpha \hbar \omega}{4}
$$

and setting $\alpha=\frac{4}{3}$ we obtain the ground state energy as

$$
E\left(\omega^{\prime}\right)=\frac{3}{4} \hbar \omega
$$

we recover the exact result (27) for both the cut-off and anharmonic oscillators because the trial wave function has the correct functional dependence on $\mathrm{r}$, when the exact functional form is unknown as observed ${ }^{1}$, one can still use the information provided by the variational principle, when the ground state should be real and non-negative and the information should be incorporated into the trial wave function and expressed in the form

$$
\psi(r)=f(r) \equiv e^{-\phi(r)}
$$

with $\phi(r) \in R$, however, since the ground eigen function must be non-degenerate, we assure that $\phi(r) \equiv \psi(r)$ for the duration to have a zero angular momentum.

\section{Conclusion}

We have demonstrated the method and techniques of variational principle to derive the ground state energy for the harmonic, cut-off and anharmonic oscillators with a ground state wave function for a one-body Hamiltonian in three dimensions. Our result shows that the variational method is appropriate in determining the exact solutions of any physical problems. Unlike the result obtained $b^{1}{ }^{1}$, our choice of the trial function leads to a generalized ground state eigen value which reduces to exact result when some parameter is adjusted. The generalized ground state eigen value reduces to exact result when some 
parameter is adjusted. The generalization of our ansatz, equation (33) and the result equation (39) there of, to study the zero point energy of a system had been investigated. Finally, the extension of the result, equation (39) to evaluate the partition function of the Bose-Einstein system is in progress.

\section{References}

1. Mur-Petit J, Polls A and Mazzanti F, Am J Phys., 2002, 70, 8.

2. Gasiorowick S, Quantum Physics, John Wiley \& Sons Inc. Toronto, Canada, 1974

3. Mavranatis H, Exercises in Quantum Mechanics, Kluwer Academia Publishers, Dordrecht, Netherlands, 1992.

4. Gottfried K, Quantum Mechanics, Addison - Wesley Reading, New York, 1989.

5. Janke W, Pelstr A, Schmidt $\mathrm{H} \mathrm{J}$ and Bachman M, Fluctuating Paths and Fields, World Scientific, Singapore, 2001.

6. Kleinert H, Path Integrals in Mechanics, Statics and Polymers Physics, World Scientific, Singapore, 1995.

7. Feynman R P and Hibs A R, Quantum Mechanics and Path Integrals, McGraw-Hill, 1965.

8. Galindo A and Tarrach R, Am J Phys., 1987, 173, 430.

9. Cohen - Tannousji C, Diu B and Laloc F, Quantum Mechanics, Wiley, New York, 1992.

10. Feennberg E, Theory of Quantum Fields, Academic, New York, 1996.

11. Gray, C G and Karl G, Progress in classical and Quantum Variational Principles, arxiv. Physics / 0512071v1 [ Physics. Class. Ph], 11 Dec, 2003.

12. Nesbet, R K, Variational Principles and methods in Theoretical Physics and Chemistry, New York, Cambridge University Press, 2003.

13. Van Brunt B, The Calculus of Variations, New York, Springer, 2004.

14. Grigorescu M, Variational Principle for Classical-Quantum Systems; arxiv: quantumph/0610011v1, 2 Oct, 2006. 


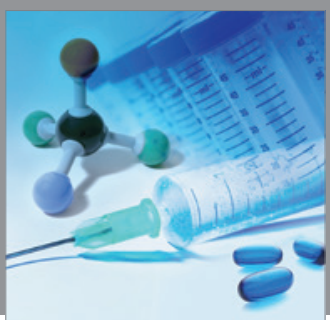

International Journal of

Medicinal Chemistry

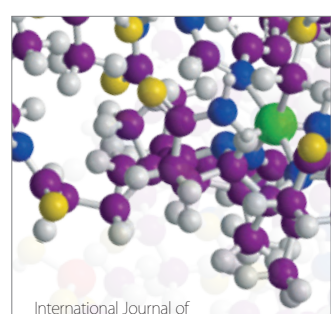

Carbohydrate Chemistry

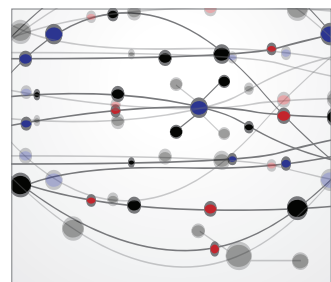

The Scientific World Journal
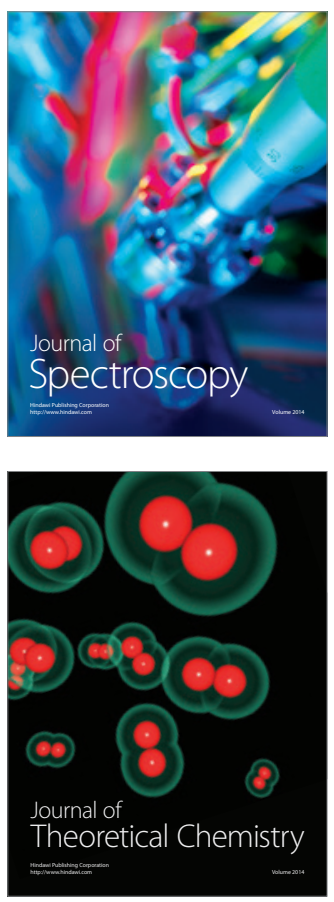
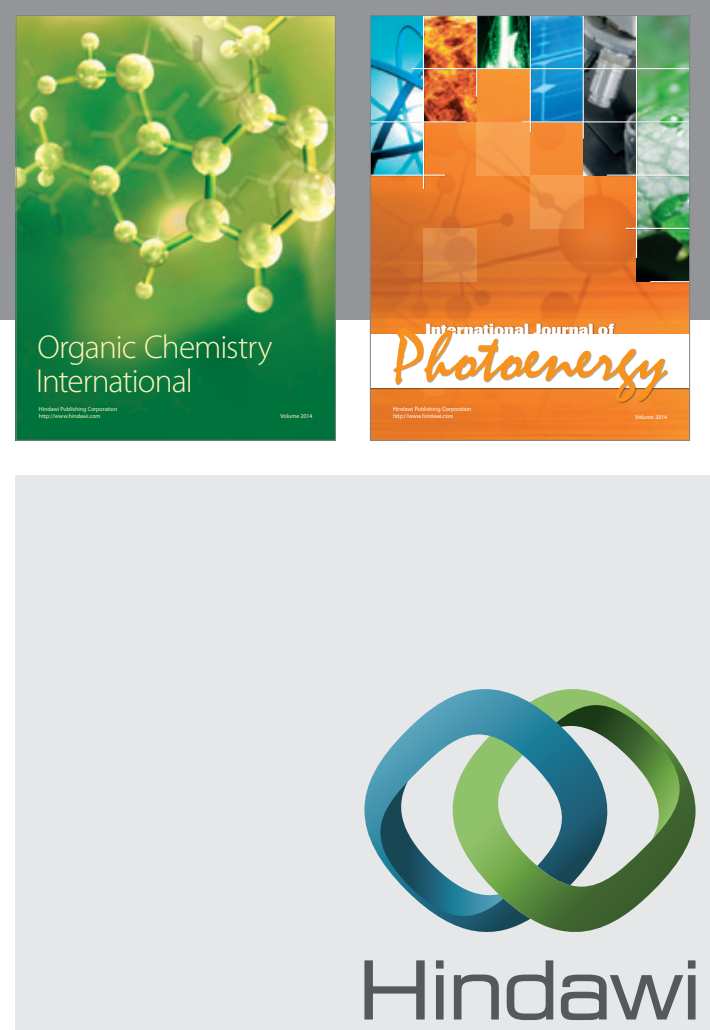

Submit your manuscripts at

http://www.hindawi.com
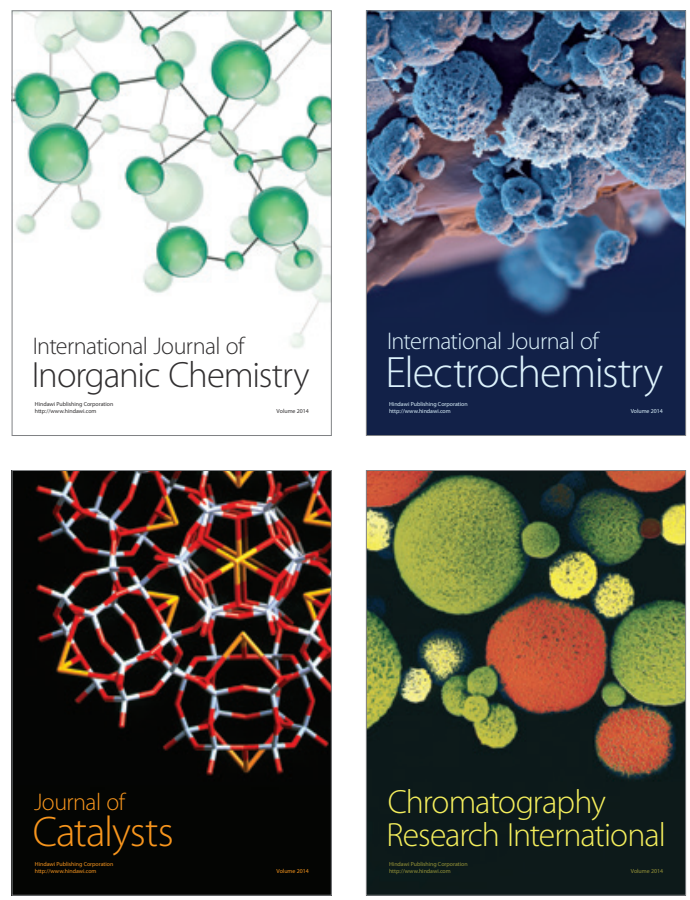
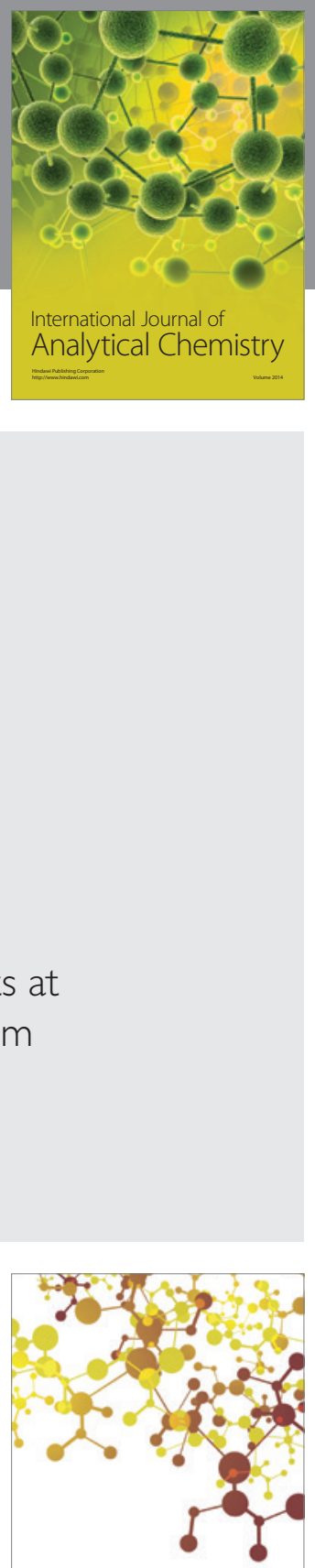

Journal of

Applied Chemistry
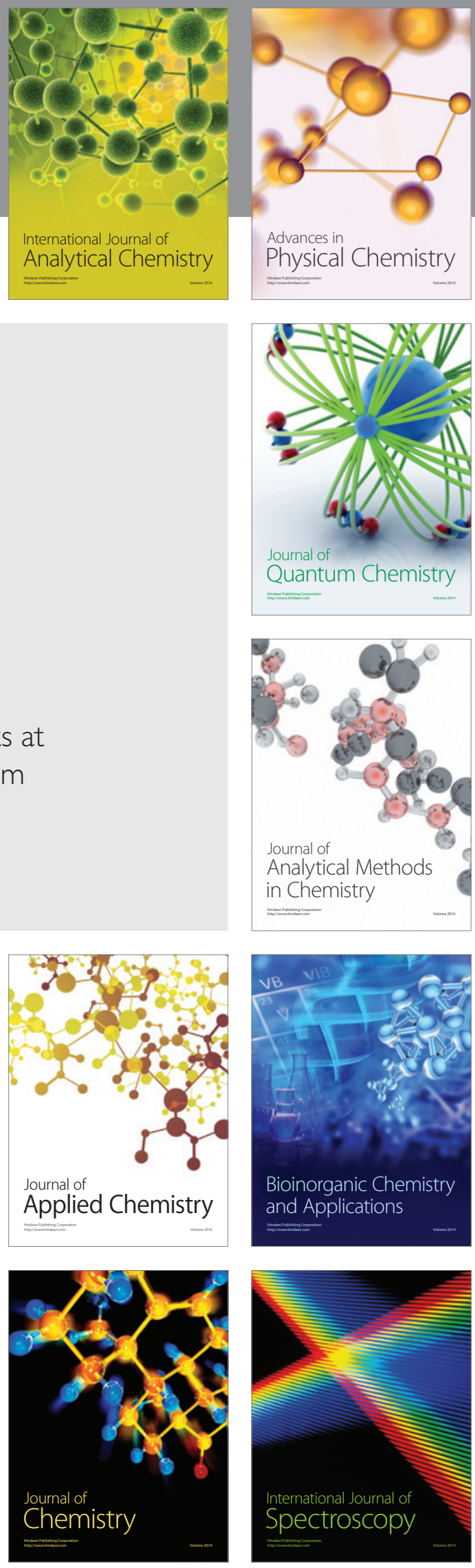